Classification

Physics Abstracts

68.55.Jk $-73.61 . \mathrm{Ga}-81.15 . \mathrm{Lm}$

\title{
Heteroepitaxy of Chemically Deposited CdS on Large Lattice Mismatched (111) GaP
}

\author{
Daniel Lincot $\left({ }^{1}\right)$, Bandombele Mokili $\left({ }^{1}\right)$, Robert Cortès $\left({ }^{2}\right)$ and Michel Froment $\left({ }^{2}\right)$ \\ $\left({ }^{1}\right)$ Laboratoire d'Électrochimie et de Chimie Analytique, Unité associée au CNRS, \\ École Nationale Supérieure de Chimie de Paris, 11 rue Pierre et Marie Curie, \\ 75231 Paris Cedex 05, France \\ $\left({ }^{2}\right)$ Physique des Liquides et Électrochimie, Unicé Propre du CNRS 15, \\ Université Pierre et Marie Curie, 4 place Jussieu, 75252 Paris Cedex 05, France
}

(Received July 16; accepted November 29, 1996)

\begin{abstract}
Heteroepitaxial CdS films $(60 \mathrm{~nm})$ have been deposited at $85^{\circ} \mathrm{C}$ on $\mathrm{GaP}$ from aqueous solutions of ammonia containing cadmium ions and thiourea precursors. Structural characterizations have been performed with RHEED, five circle XRD and TEM observations of cross sections. The large lattice mismatch results in the formation of a large density of stacking faults in the CdS leading to a polytype structure with an equal proportion of cubic and hexagonal modifications. Changes in the lattice parameter are observed in the CdS near the interface.
\end{abstract}

\section{Introduction}

Thin films of chalcogenide compounds can be deposited from chemical reactions between dissolved precursors in solutions ([1-3], and references therein). By analogy with the corresponding techniques in the vapor phase (CVD, OMCVD) this process is called chemical bath deposition (CBD). In the case of sulfides, metal complex ions and molecular sulfide precursors as thiourea or thioacetamide are mostly used. For CdS, a widely used overall reaction is:

$$
\mathrm{Cd}\left(\mathrm{NH}_{3}\right)_{4}^{2+}+\mathrm{SC}\left(\mathrm{NH}_{2}\right)_{2}+2 \mathrm{OH}^{-} \rightarrow \mathrm{CdS}+4 \mathrm{NH}_{3}+\mathrm{CN}_{2} \mathrm{H}_{2}+2 \mathrm{H}_{2} \mathrm{O}
$$

where ammonia is used as a complexing agent for cadmium ions. The deposition takes place at low temperature depending on the solution composition, typically $40-90^{\circ} \mathrm{C}$. Depending on the conditions, the growth proceeds via an heterogeneous atom by atom mechanism on the substrate or via an aggregation mechanism involving colloids formed by an homogeneous reaction in the bulk of the solution [1-3]. Thanks to superior performances, CBD has already replaced vapor phase techniques for the fabrication of CdS films in high efficiency and large area thin film solar cells based on $\mathrm{CdTe}$ and $\mathrm{CuInSe}_{2}$ absorbers. Recent papers indicate that superior performances 
may be also reached for the passivation of optoelectronic devices [4]. The use of this technique for the fabrication of epitaxial semiconductor heterostructures is thus an open field, offering noticeable advantages with respect to other techniques as MBE or MOCVD [5, 6]. They result from the very low deposition temperatures (preventing interdiffusion across interfaces) and the excellent coverage properties at low thicknesses (due to interface properties of the solids in contact with solutions) and large area capabilities. Heteroepitaxial growth of CdS has been recently demonstrated on InP substrates in growth conditions corresponding to the atom by atom mechanism

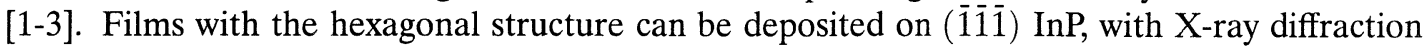
peaks presenting a full width at half maximum (FWHM) of $3.6^{\circ}$ for CdS $[1,2]$. Metastable cubic films with even a better quality are deposited on (100) InP with a FWHM of only $0.6^{\circ}$ (to be compared with to $0.24^{\circ}$ for the substrate) [3].

In the case of InP, heteroepitaxial processes are greatly favored since the lattice matching between CdS and InP is excellent $\left(f=\left(a_{\mathrm{CdS}}-a_{\mathrm{InP}}\right) / a_{\mathrm{InP}}=-0.07 \%\right)$. An important extension is to investigate the possibility of growing epitaxial films by this method when the lattice mismatch is larger. This has been done successfully in the case of MBE deposited CdS on GaAs for which $f=2.7 \%$ [7]. On the other hand epitaxial CBD-CdSe films were grown on InP with $f=2 \%$ [8].

In this paper we report the successful epitaxy of $\mathrm{CdS}$ on $\mathrm{GaP}$ substrates which present an even larger lattice mismatch, with $f=7 \%$. We have also chosen GaP since it belongs to the same series as InP, keeping in both cases the same phosphorus chemical environment for initial interfacial processes. No very recent report on the epitaxy of $\mathrm{CdS}$ on $\mathrm{GaP}$ seems to have been published to our knowledge. Previous works in this field concern epitaxial CdS layers deposited from the vapor phase at much higher temperatures $\left(300-600^{\circ} \mathrm{C}\right)$ [9-11]. The fabrication of $\mathrm{CdS} / \mathrm{GaP}$ heterostructures is basically interesting in the field optoelectronic devices based on wide gap semiconductors operating in the UV range.

\section{Experimental}

The CdS films were deposited from ammonia solutions using thiourea as the sulfur source and cadmium complex ions [1-3]. We have used a standard recipe leading to epitaxial growth on InP substrates [3]. The concentrations were as follows: ammonia $0.4 \mathrm{M}$, ammonium acetate $0.02 \mathrm{M}$, cadmium acetate $10^{-3} \mathrm{M}$, thiourea $5 \times 10^{-3} \mathrm{M}$. The deposition temperature was $85^{\circ} \mathrm{C}$. The $\mathrm{GaP}$ substrates were single crystal wafers from Rhilips (Eindhoven), kindly donated by Dr. F. Iranzo-Marin and Prof. J. Kelly of the Debye Institute, Utrecht. They were cut along (11i1). Prior to the deposition they were etched in bromine in methanol (1\% in volume) for 30 $\mathrm{s}$, then rinsed in methanol and dipped in $3 \mathrm{M}$ sulphuric acid solution for five minutes in order to remove the oxide layer. The GaP substrate was finally thoroughly rinsed in deionized water before its introduction in the reaction vessel. The volume of the solution is around $25 \mathrm{~cm}^{3}$. The deposition duration was generally $15 \mathrm{~min}$, starting from room temperature, leading to a thickness of CdS of about $60 \mathrm{~nm}$ (growth rate: $0.2-0.25 \mathrm{micron} / \mathrm{hour}$ ).

The film thickness was determined by Rutherford back-scattering (RBS) measurements. Preliminary indications on the epitaxy were obtained thanks to reflexion of high-energy electron diffraction (RHEED). X-ray diffraction allowed a quantitative determination of the structure and of the epitaxial orientation of the films. Measurements were taken using a five circle XRD goniometer specially designed for thin films studies. A Cu K $\alpha$ source, a LiF flat monochromator crystal and a NaI(Tl) detector associated with a discriminator pulse high analyser were used. The entrance slit $\left(1 \times 0.05 \mathrm{~mm}^{2}\right)$ vertically defines a very narrow beam and allows to impige a $10 \mathrm{~mm}$ of diameter sample illumination under a glancing angle. With a glancing angle of $0.6^{\circ}$, the X-ray path in the film is enhanced by a factor of 100 , so that the X-ray absorption in the film is about $50 \%$ for a $50 \mathrm{~nm}$ film thickness. The goniometer was controlled by a microcomputer which measured 
a)

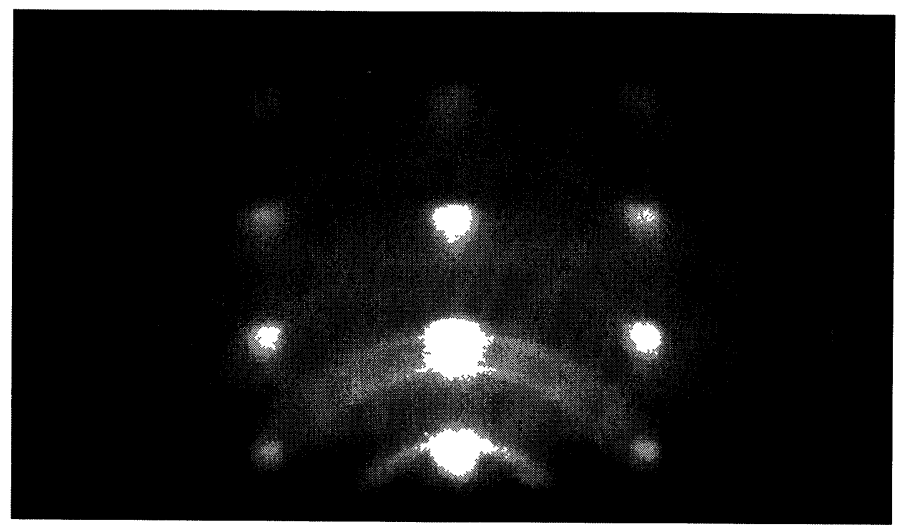

b)

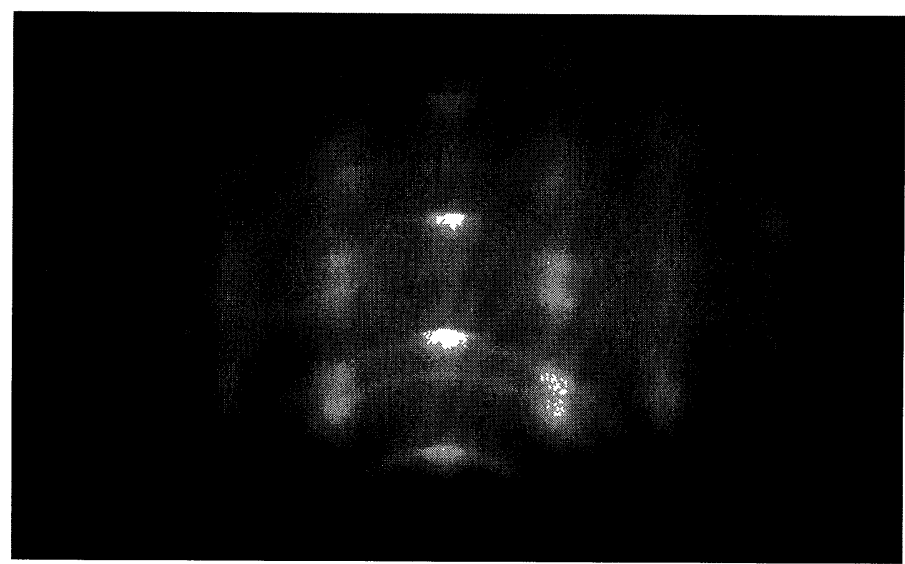

Fig. 1. - RHEED patterns of a CdS film (thickness $64 \mathrm{~nm}$ ) chemically deposited on (1̄ī) GaP. a) Observation in the $\langle 112\rangle$ azimuth in the cubic phase. b) Observation in the $\langle 1 \overline{1} 0\rangle$ azimuth in the cubic phase; presence of reflexions related to (111) twins.

the position and the intensities of selected Bragg peaks. For investigating the sample crystallinity, two kinds of scans were mainly used: (i) with a glancing angle incidence, a $\Psi$ scan around an axis normal to the sample surface; and (ii) in the condition $\theta-2 \theta$ around an axis parallel to the sample surface and normal to the X-ray beam. The structure of the interface was studied thanks to high resolution transmission electron microscopy observations (HREM) of cross sections using a Philips CM 20 apparatus operated at $200 \mathrm{kV}$. Thin foils were prepared by ion milling. Scanning electron microscopy observations have been used to inspect the surface morphology of the CdS deposits.

\section{Results and Discussion}

Figure 1 shows RHEED patterns obtained for a CdS film deposited on the (1̄îj) face of GaP. They correspond to typical patterns of monocrystalline CdS with a weak contribution of polycrystalline CdS. Figure 1a could be indexed as cubic ( $\langle 112\rangle$ azimuth) or hexagonal ( $\langle 1 \overline{1} 0\rangle$ azimuth) $\mathrm{CdS}$. Figure $1 \mathrm{~b}$, if indexed as cubic $(\langle 1 \overline{1} 0\rangle$ azimut) shows a high density of twins. The broadening of the spots observed in Figure 1 is consistent with the values of the FWHM found on the XRD 


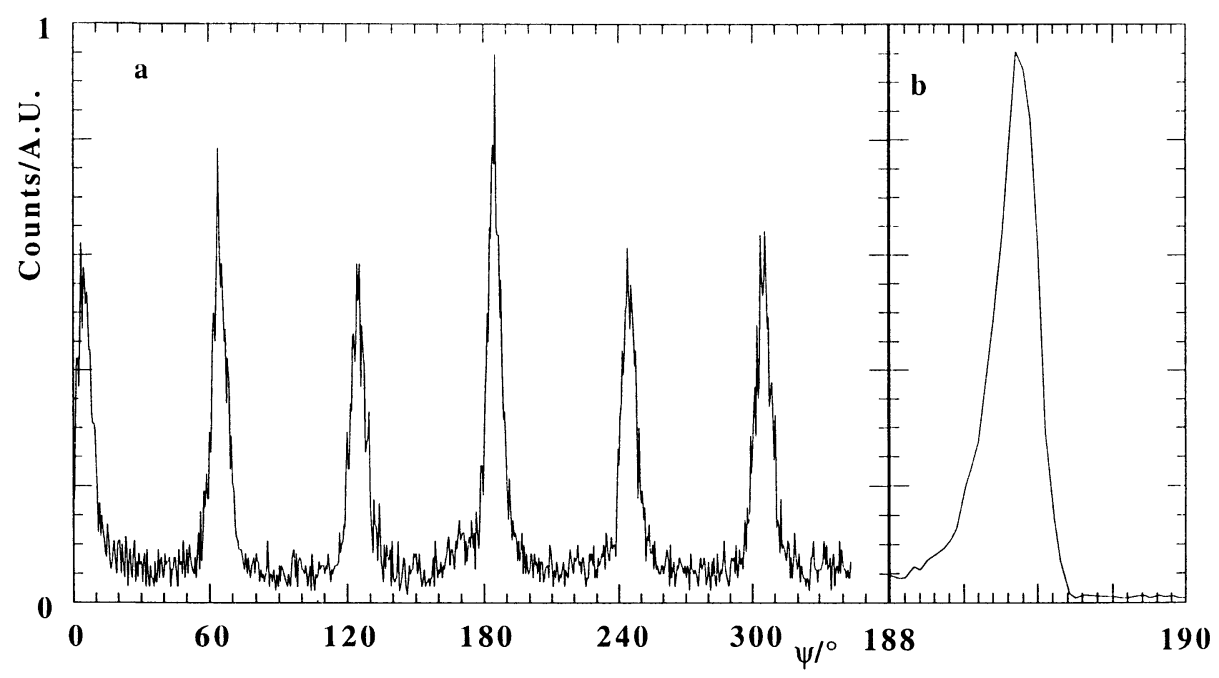

Fig. 2. - XRD diagram of an epitaxial CdS film (thickness $64 \mathrm{~nm}$ ) chemically deposited on (īīi) GaP. a) (10i1) reflexions of the hexagonal CdS phase. b) (220) reflexion of the GaP substrate.

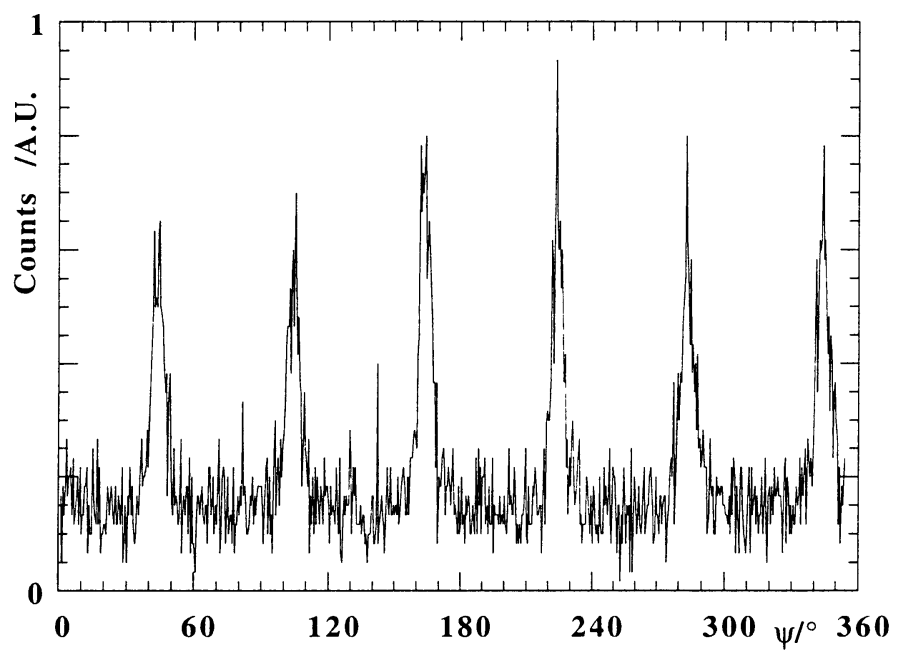

Fig. 3. - XRD diagram of an epitaxial CdS film (thickness $64 \mathrm{~nm}$ ) deposited on (īi 1 ) GaP. Observation of six (111) reflexions of the cubic phase.

peaks (see below). The broadening of the spots is not connected to surface contamination because the aspect of the RHEED patterns is not modified when the reflexion angle is changed. In order to investigate the $\mathrm{CdS}$ structure, $\mathrm{XRD} \Psi$ scans were taken with a $0.6^{\circ}$ angle. We observed respectively the CdS (1011) reflexions which are characteristic of the hexagonal phase (Fig. 2) and the CdS (111) reflexions which are characteristic of the cubic phase (Fig. 3). This comes from the polymorphism of CdS, the hexagonal modification being only slightly more stable than the cubic one. The conditions to observe these reflexions have been chosen in order to select peaks far from those of the substrate. Measurements of the area of the peaks show that the epitaxial CdS is 


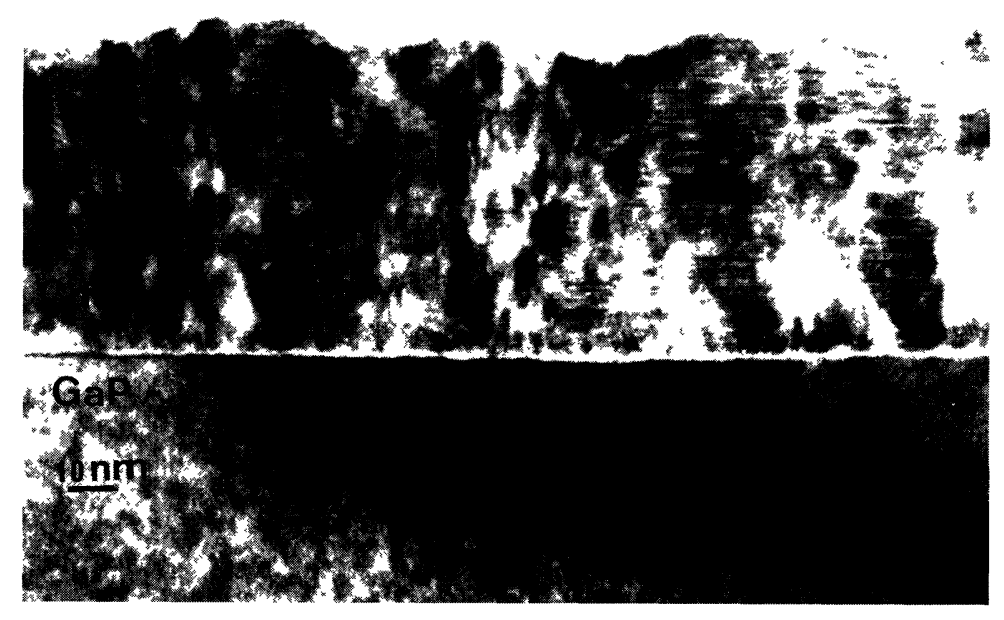

Fig. 4. - TEM images of a cross sectional thin foil prepared from a CdS/GaP sample. a) Observation of planar defects in a low magnification image. b) Lattice image of the CdS/GaP interface.

a mixture of the hexagonal and the cubic phase in about the same proportion. For the cubic phase (Fig. 3) six peaks are observed instead of three as expected. This can be explained by the presence of twins in the CdS layer. It occurs because two different structural (111) arrangements of $\mathrm{CdS}$ may exist, with a $180^{\circ}$ rotation between them. The CdS peaks have a full width at half maximum (FWHM) of $8^{\circ}$ for the hexagonal phase (Fig. 2a) and a similar value of $7^{\circ}$ for the cubic phase (Fig. 3). These values are significantly broader than the FWHM of the GaP (220) reflexion, as shown in Figure $2 \mathrm{~b}\left(\mathrm{FWHM}=0.3^{\circ}\right)$. Thus the cristallinity is not as good as that obtained $\left(\mathrm{FWHM}=3.6^{\circ}\right)$ by chemical deposition of CdS on $\operatorname{InP}(\overline{1} \overline{1} \overline{1})[1,2]$. However in this case the lattice mismatch is much smaller $(f=-0.07 \%)$ than for the epitaxial growth of CdS on GaP $(f=7 \%)$. In the same way $\theta-2 \theta \mathrm{X}$-ray scans give a larger peak $\left(4^{\circ}\right)$ than that relative to the substrate $\left(0.5^{\circ}\right)$.

A TEM image of a cross-sectional thin foil prepared from a CdS/GaP sample is shown in Figure 4. This low magnification image reveals a high density of planar defects parallel to the interface. This image also shows that the CdS layer presents a strong corrugation. SEM observations (Fig. 5) of the CdS surface confirm the presence of a high density of pyramids $\left(10^{9} \mathrm{~cm}^{-2}\right)$. Figure 6 is a lattice image where the GaP [110] axis is aligned along the electron beam direction. In some places the interface is well resolved and misfit dislocations can be detected. In other places the interface is more disturbed. It seems that the disturbed zone, in the center of Figure 6 , corresponds to the superposition of two domains located respectively on the left and on the right and exhibiting different staking sequences. This phenomenon is probably related to the important stresses coming from the large mismatch between $\mathrm{CdS}$ and $\mathrm{GaP}$. Chemical reactions between the solution and GaP, just after the immersion, cannot be excluded, as compared to the results obtained with sulfide treatments on InP $[12,13]$ where the formation of an interfacial indium sulfide layer is shown. In our case, after the growth of 3-5 CdS layers, high resolution images are observed. Nevertheless the CdS layer contains a lot of dislocations, stacking faults and microtwins. Consequently during the growth process successive areas exhibiting an hexagonal or cubic stacking are formed [14]. This observation is in agreement with the existence of X-ray diffraction $\Psi$ scans (Figs. 2,3 ) corresponding to the formation of epitaxial hexagonal and cubic CdS with almost the same FWHM. 


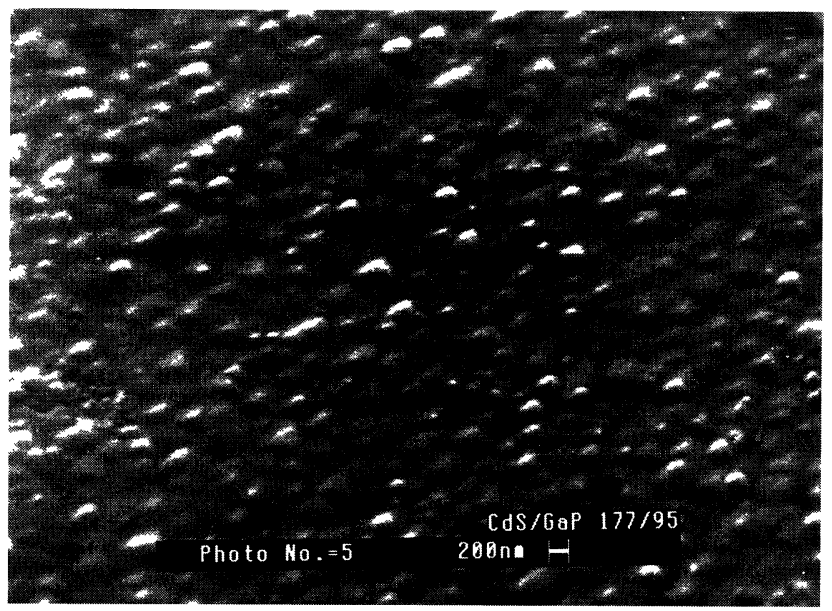

Fig. 5. - SEM image showing pyramids at the surface of a CdS epitaxial film grown on (īīi) GaP.

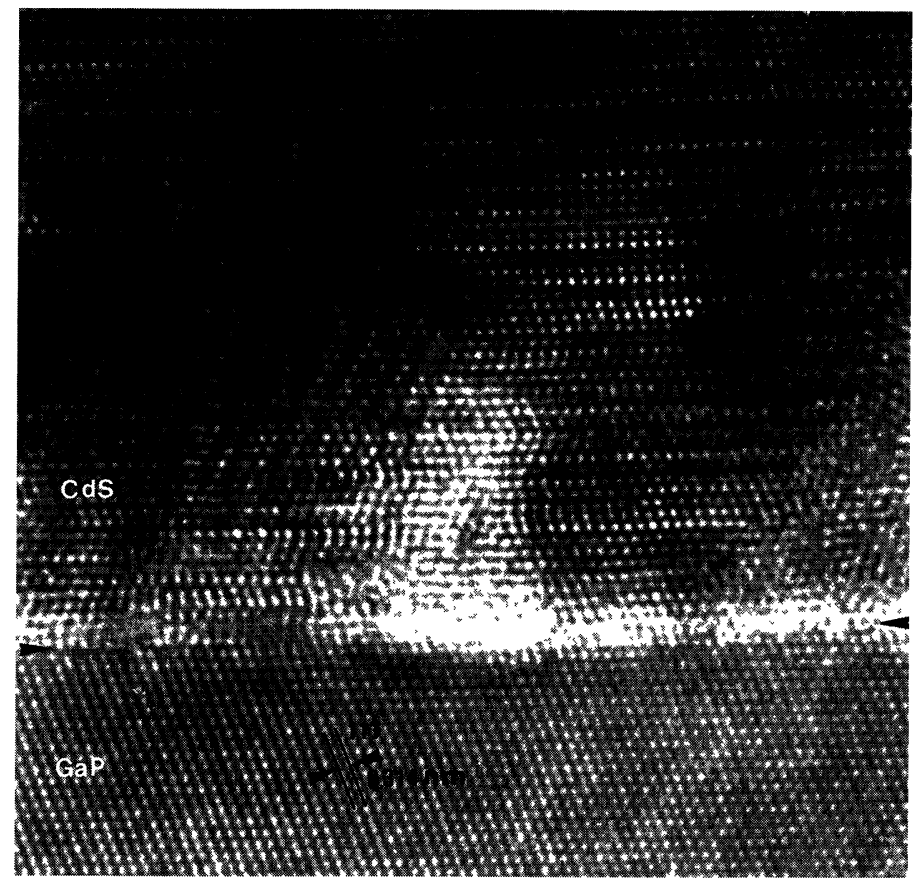

Fig. 6. - Lattice image of the $\mathrm{CdS} / \mathrm{GaP}$ interface.

On the other hand measurements of the interplanar spacing on HREM photographies strongly enlarged (using ten successive interplanar distances) reveal some variations from the interface to the bulk deposit. A decrease of the interplanar spacing is peculiarly observed for the (111) plane crossing the interface with an angle of $70^{\circ}$ (as indicated in Fig. 6). Near the interface the $d_{(111)}$ spacing is $0.345 \pm 0.003 \mathrm{~nm}$ and decreases to $0.336 \pm 0.003 \mathrm{~nm}$ at about $10 \mathrm{~nm}$ in the CdS. 
This value is close to the $(111)_{\mathrm{c}}$ spacing $(0.3357 \mathrm{~nm})$. Such a variation is less important for the (111) planes parallel to the interface. It is interesting to recall the results obtained by Sinha $e t$ al. [7]. For CdS on GaAs $(f=2.7 \%)$. They found that the accommodation of the lattice mismatch was done by a continuous distribution of compressive stress in the CdS layer (parallel to the interface plane) which extends from the interface to about $15 \mathrm{~nm}$ in the layer associated to concomitant variation of the lattice parameter. As a consequence one can anticipate that the change of the lattice parameter in this case is also related to strain relaxation. It is surprising that the variation is in opposite direction to that expected in the interface plane from the lattice mismatch: $a(\mathrm{CdS})>a(\mathrm{GaP})$. This could be a consequence of the formation of an interfacial foreign layer. In the present case, from a structural point of view, a $\mathrm{GaPS}_{4}$ layer could be formed. This compound has a structure which can be derived from a hexagonal close-packed arrangement of the sulphur atoms [15]. It presents a (021) interreticular distance of $0.352 \mathrm{~nm}$ which is close to the value $(0.345 \mathrm{~nm})$ found near the interface.

The other important result from this study is that the CdS layer is not purely hexagonal or cubic but a mixture between both modifications with almost the same proportions. The intimate and regular lamellae organization shown in HREM images suggest that the final structure corresponds to a polytype structure, with a short length cell unit. Polymorphism is characteristic of compounds which present different modifications with only little difference in energy as $\mathrm{ZnS}, \mathrm{CdS}$ and $\mathrm{CdSe}$. For $\mathrm{CdS}$ the hexagonal phase $(2 \mathrm{H})$ is slightly more stable than the cubic phase (3C) $(-1.1 \mathrm{meV} / \mathrm{atom})[16]$. Polytypes are metastable phases which are also intermediate in the phase transformation processes [17]. Short period polytypes have been identified in $\mathrm{CdS}$ as $n \dot{\mathrm{H}}(4 \mathrm{H}$, $5 \mathrm{H}, 6 \mathrm{H})$, where $n$ denotes the number of atomic planes in the unit cell with cubic and hexagonal stacking and $\mathrm{H}$ the hexagonal modification [18]. In $4 \mathrm{H}$, the proportion cubic to hexagonal is $50 \%$ as for our results. Similar polytype structures have been identified in CdSe clusters [19]. An interesting point is to determine if the polytype structure is related or not to the mechanism of lattice mismatch accommodation. Previous results [2,3] obtained on InP for the same deposition conditions of $\mathrm{CdS}$ show that on ( $(\overline{1} \overline{1} \overline{1})$ InP the hexagonal phase is majoritary in the film with much lower densities of stacking faults. On (100) InP the cubic phase, with a very small defect density, is formed. On silicon or (111) InP polycrystalline films are formed. This means that the structure of the film cubic or hexagonal is determined by substrate effects. This is an argument to associate the polytype structure obtained in the case of $\mathrm{GaP}$ also to substrate effects and thus to the large lattice mismatch. One can note that in the epitaxial growth of CdTe on CdS, recently obtained by electrodeposition [20], a large lattice mismatch exists between $\mathrm{CdS}$ and $\mathrm{CdTe}(f=-10.3 \%)$. In that case also, the epitaxy was shown to be possible but the presence of stacking faults parallel to the substrate was observed. On the other hand the large $\theta-2 \theta \mathrm{X}$-ray peaks could be interpreted by the high density of stacking faults and by the variations of the lattice interplanar distances.

The large mismatch between $\mathrm{CdS}$ and $\mathrm{GaP}$ is not only responsible for the formation of misfit dislocations and stacking faults in the epitaxial layers [21,22] but the resulting stresses can also explain the roughening of the epilayers observed in Figures 5 and 6 . This relation has been discussed recently [23].

\section{Conclusion}

In this paper we have demonstrate that epitaxial films of $\mathrm{CdS}$ on GaP can be prepared at low temperature by CBD despite a lattice mismatch of $7 \%$. Strain relaxation at the interface may be responsible for the formation of a high density of stacking faults parallel to the substrate. The resulting structure of the layer is in fact a mixed cubic-hexagonal polytype structure. These results 
suggest that the chemical bath deposition may be a good technique to prepare heteroepitaxial structures between III-V and II-VI compounds with a good accommodation of large lattice mismatchs.

\section{Acknowledgements}

We gratefully acknowledge B. Jouffrey for the use of the CM 20 electron microscope (École Centrale, Chatenay Malabry). Mrs Françoise Pillier is acknowledged for the sample preparations.

\section{References}

[1] Lincot D., Ortega-Borges R. and Froment M., Appl. Phys. Lett. 64 (1994) 569.

[2] Froment M., Bernard M.C., Cortes R., Mokili B. and Lincot D., J. Electrochem. Soc. 142 (1995) 2642.

[3] Cortès R, Froment M., Mokili B. and Lincot D., Philos. Mag. Lett. 73 (1996) 209.

[4] a) Vaccaro K., Dauplaise H.M., Davis A., Spaziani S.M. and Lorenzo J.P., Appl. Phys. Lett. 67 (1995) 2873; b) Vaccaro K, Davis A., Dauplaise H.M., Spaziani S.M., Martin E.A. and Lorenzo J.P., J. Electron. Mater. 25 (1996) 603.

[5] Wilke W.G., Seedorf R. and Horn K., J. Vac. Sci. Technol. B7 (1989) 807.

[6] Schultz Ch., Von Der Emde and Lahn D.R.T., Phys. Status Solidi A 145 (1994) 497.

[7] Sinha K., Menéndez J., Niles D.W. and Höchst H., J. Vac. Sci. Technol. B9 (1991) 2202.

[8] Cachet H., Froment M. and Maurin G., J. Electroanal. Chem. 406 (1996) 239.

[9] Weinstein M.and Wolff G.A., J. Phys. Chem. Solids Suppl. 1 (1967) 537.

[10] Kotelyanskii I.M., Mityagin A. Yu. and Orlov V.P., J. Crystal Growth 10 (1971) 191.

[11] Kuznetsov P.I., Shemet V.V., Odin I.N. and Novosalova A.V., Izvestiya Akademii Nauk SSSR, Neorganicheskie Materialy 17 (1981) 791.

[12] Iyer R., Chang R.R. and Lile D.L., Appl. Phys. Lett. 53 (1988) 134.

[13] Wilmsen C.W., Geib K.M., Shin J. Lyer R. Lile D.L. and Pouch J.J., J. Vac. Sci. Technol. B7 (1989) 851.

[14] Froment M. and Lincot D., Electrochim. Acta. 40 (1995) 1293.

[15] Buck P. and Carpentier C.D., Acta Cryst. B29 (1973) 1864.

[16] Yeh C.Y., Lu Z.W., Froyen S. and Zunger A., Phys. Rev. B 46 (1992) 10086.

[17] Engel G.E., Needs R.J., J. Phys. Condens. Matter 2 (1990) 367 and 6905.

[18] Prasad S., Tiwari B.K. and Srivastava O.N., Acta Cryst. A 37 (1981) 442.

[19] Bawendi M.G., Kortan A.R., Steigerwald M.L. and Brus L.E., J. Chem. Phys. 91 (1989) 7282.

[20] Lincot D., Mokili B., Kampmann A., Vedel J., Cortès R. and Froment M., Appl. Phys. Lett. 67 (1995) 2355.

[21] Ruvimov S., Bourret E.D., Washburn J. and Lilienthal-Weber Z., Appl. Phys. Lett. 68 (1996) 346.

[22] Bourret-Courchesne E.D., Appl. Phys. Lett. 68 (1996) 1675.

[23] Jesson D.E., Chen K.M. and Pennycook S.J., MRS Bull. (April, 1996) p. 31. 